\title{
Diagnostic accuracy of quantitative micro-elastography for margin assessment in breast- conserving surgery
}

Kelsey M. Kennedy ${ }^{1 *}$, Renate Zilkens ${ }^{1,2}$, Wes M. Allen ${ }^{1,3}$, Ken Y. Foo ${ }^{1,3}$, Qi Fang ${ }^{1,3}$, Lixin Chin ${ }^{1,3}$, Rowan W. Sanderson ${ }^{1,3}$, James Anstie ${ }^{1,3}$, Philip Wijesinghe ${ }^{1,3, \dagger}$, Andrea Curatolo ${ }^{1,3, \neq}$, Hsern Ern I. Tan $^{2}$, Narelle Morin ${ }^{4}$, Bindu Kunjuraman ${ }^{5}$, Chris Yeomans ${ }^{6}$, Synn Lynn Chin ${ }^{5}$, Helen DeJong ${ }^{1}$, Katharine Giles ${ }^{7}$, Benjamin F. Dessauvagie ${ }^{2,6}$, Bruce Latham ${ }^{6}$, Christobel M. Saunders ${ }^{2,5,8}$, and Brendan F. Kennedy ${ }^{1,3}$

${ }^{1}$ BRITElab, Harry Perkins Institute of Medical Research, QEII Medical Centre, Nedlands, and Centre for Medical Research, The University of Western Australia, Perth, Australia

${ }^{2}$ School of Medicine, The University of Western Australia, Perth, Australia

${ }^{3}$ Department of Electrical, Electronic \& Computer Engineering, School of Engineering, The University of Western Australia, Perth, Australia

${ }^{4}$ Sonowest, Perth, Australia

${ }^{5}$ Breast Centre, Fiona Stanley Hospital, Murdoch, Australia

${ }^{6}$ PathWest, Fiona Stanley Hospital, Murdoch, Australia

${ }^{7}$ OncoRes Medical, Perth, Australia

${ }^{8}$ Breast Clinic, Royal Perth Hospital, Perth, Australia

${ }^{*}$ Current affiliation: Department of Biomedical Engineering, Columbia University, New York, NY

${ }^{\dagger}$ Current affiliation: School of Physics and Astronomy, University of St Andrews, United Kingdom ${ }^{\ddagger}$ Current affiliation: VioBio, Instituto de Óptica "Daza de Valdés", Consejo Superior de Investigaciones Cientificas (IO, CSIC), Madrid, Spain

Running title (60 characters): Quantitative micro-elastography of breast tumor margins

Keywords: breast-conserving surgery, surgical guidance, tumor margins, elastography

Financial support: The authors acknowledge support from OncoRes Medical; Department of Health, Australia; Cancer Council Western Australia; Australian Research Council

Corresponding author: Brendan F. Kennedy, +61 6151 1085, brendan.kennedy@uwa.edu.au

Conflict of interest: This work received funding from OncoRes Medical Pty Ltd, in which KMK, $\mathrm{LC}, \mathrm{AC}, \mathrm{BL}, \mathrm{CMS}$ and BFK disclose financial interest.

Manuscript length: 5370 words; 4 figures, 3 tables

\begin{abstract}
Inadequate margins in breast-conserving surgery (BCS) are associated with an increased likelihood of local recurrence of breast cancer. Currently, approximately $20 \%$ of BCS patients require repeat surgery due to inadequate margins at the initial operation. Implementation of an accurate, intraoperative margin assessment tool may reduce this re-excision rate. This study determined, for the first time, the diagnostic accuracy of quantitative micro-elastography (QME), an optical coherence tomography (OCT)-based elastography technique that produces images of tissue micro-scale elasticity, for detecting tumor within $1 \mathrm{~mm}$ of the margins of BCS specimens. Simultaneous OCT and QME were performed on the margins of intact, freshly excised specimens from 83 patients undergoing BCS and on dissected specimens from 7 patients undergoing mastectomy. The resulting three-dimensional images $(45 \times 45 \times 1 \mathrm{~mm})$ were co-registered with post-operative histology to determine tissue types present in each scan. Data from 12 BCS patients and the 7 mastectomy patients served to build a set of images for reader training. 154
\end{abstract}


sub-images $(10 \times 10 \times 1 \mathrm{~mm})$ from the remaining 71 BCS patients were included in a blinded reader study, which resulted in $69.0 \%$ sensitivity and $79.0 \%$ specificity using OCT images, versus $92.9 \%$ sensitivity and $96.4 \%$ specificity using elasticity images. The quantitative nature of QME also facilitated development of an automated reader, which resulted in $100.0 \%$ sensitivity and $97.7 \%$ specificity. These results demonstrate high accuracy of QME for detecting tumor within $1 \mathrm{~mm}$ of the margin and the potential for this technique to improve outcomes in BCS.

\section{Introduction}

Success of breast-conserving surgery (BCS) is characterized by clear margins and a good cosmetic outcome for the patient. However, $\sim 20 \%$ of cases require repeat surgery due to inadequate margins (1-3). Re-excision surgery causes substantial physical, psychological, and financial burdens for patients, with higher risk of complications (4), worse cosmesis, and additional costs, on average, of $>\$ 10,000$ per patient (5). Intraoperative detection of tumor at the margins would allow more complete resection of malignant tissue in the first operation, provide the surgeon with confidence that no residual cancer remains in the breast, and reduce the number of reexcision surgeries.

Several techniques are currently used for intraoperative margin assessment. Intraoperative pathological assessment can be performed using frozen section analysis and imprint cytology (6), but these techniques are resource-intensive, sample only a small percentage of the surgical margins, and have limited efficacy, especially for ductal carcinoma in situ (DCIS) (7), and, thus, have not been widely adopted (8). Another available technique, intraoperative specimen radiography (IOSR), provides an X-ray image of the excised specimen. IOSR can show that the main lesion has been removed, which is particularly useful for non-palpable tumors that are localized using pre-operative wire insertion (9) or radioactive seed placement (10). However, IOSR accurately predicts margin status in only $48 \%$ of cases (11) and has been shown not to reduce re-excision rates $(11,12)$. Intraoperative ultrasound guidance of excision has been shown in a small number of studies to reduce re-excision rates by more than half for invasive cancers $(13,14)$, but ultrasound is operator-dependent and has limited reliability for visualizing in situ or multifocal cancers $(15,16)$.

To address the shortcomings of existing techniques, a range of margin assessment tools have been proposed, relying on various contrast mechanisms to detect cancer. One commercially available technique uses radiofrequency spectroscopy to measure the dielectric properties of tissue, implemented in a handheld probe (MarginProbe, Dune Medical Devices). The technique shows high accuracy for margin assessment in homogeneous, ex vivo tissues (e.g., if the probe is placed over a large region of tumor, $>6 \mathrm{~mm}$ diameter) but has lower accuracy $(70 \%$ sensitivity and $70 \%$ specificity) when multiple tissue types are present in the interrogated region (17). In a randomized trial with 596 patients, use of MarginProbe on excised specimens reduced re-excision rate from $25.8 \%$ to $19.8 \%$ but suffered from low specificity with a $53.6 \%$ false positive rate (18).

Fluorescent modalities that utilize molecular contrast to intraoperatively highlight cancer are also under development, potentially enabling surgeons to visualize tumor in the cavity, as well as in the excised lump (19-22). A small number of proof-of-principle studies in humans have been reported $(19,20,22)$, but the efficacy of these techniques for reducing re-excision rates in BCS has not been determined. In addition, they typically rely on pre-operative, systemic administration of exogenous dyes, requiring extensive dosing and tumor uptake studies, and creating potential barriers to clinical translation (19). Another optical technique, surface-enhanced Raman scattering (23), has recently been proposed, using targeted nanoparticles for multiplexed imaging of cancer biomarkers. A preliminary study on ex vivo tissues dissected from mastectomy and 
lumpectomy specimens showed promising sensitivity (89.3\%) and specificity (92.1\%) for cancer detection, and the technique is conducive to personalized biomarker imaging based on tumorspecific molecular profiles. However, it is restricted to surface imaging, and, while many institutions have adopted a "no tumor on ink" criterion for invasive cancers, larger margins are typically desired for in situ cancers (1). Label-free optical techniques have also been proposed, including diffuse reflectance spectroscopy/hyperspectral imaging (24, 25), autofluorescence lifetime imaging, and Raman spectroscopy (26). However, diffuse techniques can suffer from low spatial resolution ( $5 \mathrm{~mm}$ ) (24), while autofluorescence and Raman techniques typically have low scanning speeds (12-24 minutes per margin) (26), making clinical translation more challenging.

Optical coherence tomography (OCT) is a promising optical technique capable of threedimensional (3-D), high-speed, high-resolution imaging without need for exogenous contrast agents. OCT may be described as an optical analogue to ultrasound. It uses interferometry to effectively measure "time of flight" of light in tissue, creating an image based on the amount of back-scattered light, with microscopic resolution $(2-10 \mu \mathrm{m})$ up to 1-2 $\mathrm{mm}$ in depth. These imaging specifications match well with the clinical requirements of margin assessment in BCS. Preliminary studies have reported high sensitivity (80-94\%) and specificity (87-93\%) for detecting cancer, primarily in mastectomy tissues (27-29). However, these mastectomy samples were typically dissected to expose a bulk of dense, high-grade tumor for imaging. The image contrast in this scenario may not translate to margin assessment in BCS, in which typically lower-grade tumors must be detected at the edge of intact specimens or, for detecting residual tumor, directly within the surgical cavity. The largest study to date that used OCT to assess margins in BCS specimens reported sensitivity of $55-65 \%$ and specificity of $68-70 \%$ (30). One reason for this relatively low accuracy may be the limited ability of OCT to distinguish between tumor and surrounding normal stroma (31-33). Stronger contrast between tissue types is expected to aid surgeon decisionmaking in the intraoperative setting $(34,35)$.

Beyond its distinct molecular and optical properties, breast cancer also exhibits distinct mechanical properties (36-38). At the cellular scale, atomic force microscopy has revealed unique mechanical signatures within the breast tumor microenvironment caused by the mix of cellular proliferation and desmoplastic stroma (37). At the macro-scale, surgeons rely on mechanical changes as they manually palpate the tissue, feeling for the boundaries of the typically stiff lesion. However, palpation is a subjective tool, and a large proportion of breast lesions are considered "impalpable," that is, too small or soft to detect through touch (39). Elastography is a technique that creates images of the mechanical properties of tissue, complementing palpation by visualizing mechanical changes in 2-D or 3-D. Elastography based on ultrasound has been developed for a number of applications, including preoperative diagnosis of breast lesions (38, 40), but has not been applied to intraoperative margin assessment, mainly due to its relatively low spatial resolution (see Supplementary Information, "Note on elastography of the breast.") In an emerging technique, OCT elastography is used to measure tissue deformation under an applied load, offering 3-D maps of mechanical properties with micro-scale resolution (41). OCT elastography techniques can be classified according to the mechanical loading mechanism, with compression and shear wave being the most prominent (41). While early compression OCT elastography studies produced maps of tissue deformation (strain) $(31,42)$, which is an indirect and qualitative measure of elasticity, introduction of a stress sensing technique to map the local stress in 2-D at the tissue surface, has enabled quantitative micro-elastography (QME), providing 3-D maps of local elasticity, under the assumption of uniaxial stress (43). Preliminary QME data in mastectomy specimens showed that elasticity images provide additional contrast between tumor and normal tissue compared to OCT and strain, and the technique has been extended to incorporate a wide-field scanning mechanism that enables entire margins of BCS specimens to be imaged within an intraoperative timeframe (44). To build on these promising feasibility studies 
and to determine the clinical potential of QME for intraoperative margin assessment, it must be established whether clinicians can interpret QME images to accurately identify close or positive tumor margins in BCS specimens. To this end, the goal of this study is to conduct, for the first time, a blinded reader study, with post-operative histology as the gold standard, to determine the diagnostic accuracy (sensitivity and specificity) of QME, compared to OCT alone, for detecting tumor within $1 \mathrm{~mm}$ of the margins of freshly excised specimens from patients undergoing BCS.

\section{Methods}

\section{Patient recruitment and imaging}

All study procedures were approved by the ethics board of the South Metropolitan Area Health Service in Western Australia. 90 patients were recruited for this study: 83 patients (with no prior excision) undergoing BCS for treatment of breast cancer, and 7 patients undergoing mastectomy for treatment of breast cancer. Samples from the 7 mastectomy patients and 12 of the BCS patients were used to create a set of pilot data for training readers. Data from the remaining 71 BCS patients were included in the blinded reader study. Table 1 summarizes the disease characteristics of all BCS patients. Table S1 summarizes mastectomy patient characteristics.

For patients undergoing BCS, following surgery, the fresh, intact specimens were transferred to the pathology department at Fiona Stanley Hospital. Pathologists at this institution dictated that tissue should be placed in fixative (formalin) within 1 hour of receiving the specimen from surgery, to avoid any tissue degradation that might influence histological processing. Between excision and imaging, specimens were kept in air at room temperature, and the surface was kept hydrated by applying droplets of saline. Specimen orientation was maintained using clips and sutures, per standards at this institution. In most cases ( 8 of 12 pilot cases and 68 of 71 cases for the reader study), two margins were imaged. In the remaining cases, one margin was imaged due to delays in scanning the specimen within the allotted time. Margins for scanning were chosen based on consultation with pathologists, observation of the intraoperative specimen radiography (when available), and surgical notes indicating if extra cavity shavings were performed intraoperatively. The "closest tumor margin" distances reported in Table 1 were determined by postoperative histology and include superficial and deep margins (considering radial margins only, which are most clinically relevant, the rate of tumor margins $<1 \mathrm{~mm}$ in the reader study was $21 \%$ ). For patients undergoing mastectomy, non-diagnostic tissue was dissected by a pathologist to create samples of $\sim 5 \times 5 \times 0.5 \mathrm{~cm}$, which were also imaged within $\sim 1$ hour of excision.

Imaging was performed on a benchtop, wide-field QME system as described previously (Fig. S1A) (44). Briefly, the system is based on a Telesto II spectral-domain OCT system (TEL220C1, Thorlabs). It uses a superluminescent diode light source with a central wavelength of $1300 \mathrm{~nm}$ and a bandwidth of $>170 \mathrm{~nm}$, illuminating the sample with $2.5 \mathrm{~mW}$ of power (a low power level that is safe for users). The measured axial and lateral resolutions in air are $5.5 \mu \mathrm{m}$ and $13 \mu \mathrm{m}$, respectively. The system captures one-dimensional axial scans (A-scans) in $14 \mu \mathrm{s}$, and the beam is raster scanned to build a 3-D image measuring $16 \times 16 \times 3.5 \mathrm{~mm}$ in $55 \mathrm{~s}$. Wide-field images are generated by translating the specimen relative to the OCT scan head between 3-D acquisitions, as described previously $(42,44)$. Nine sub-volumes are acquired with 1-mm overlap in the lateral plane, resulting in a $45 \times 45 \times 3.5 \mathrm{~mm}$ image captured in under 9 minutes. The partially overlapping sub-volumes are stitched to form mosaicked wide-field images, presented in the en face plane. The measured displacement sensitivity of the OCT system is $1.4 \mathrm{~nm}$ at an OCT signal-to-noise ratio of $40 \mathrm{~dB}$, acquired under clinical testing conditions in the pathology laboratory (i.e., without a vibration isolation table). 
A compliant silicone layer is placed between the specimen and imaging window to ensure contact between the uneven specimen surface and window, as well as to estimate stress for quantification of elasticity (43). After establishing contact, micro-scale displacement (up to $9.5 \mu \mathrm{m}$ ) was applied to the specimen surface using a piezoelectric actuator. Images were processed to determine stress, strain, and elasticity (Fig. S1B), and elasticity was overlaid on the non-adipose regions of the OCT images for visualization, using an algorithm described previously (42). OCT data is displayed in grayscale from 0 to $40 \mathrm{~dB}$, and elasticity data is displayed in color on a logarithmic scale from 3.63 to $363 \mathrm{kPa}$.

\section{Histopathology and co-registration with imaging data}

Following imaging, specimens were fixed in formalin and submitted for standard histopathological processing. Specimens were inked for orientation and sliced in "bread-loaf" fashion from lateralto-medial, superficial-to-deep, or superior-to-inferior margins to generate histological sections at each plane. This sectioning protocol resulted in histology typically being performed in a plane orthogonal to the en face plane in which QME images are displayed. The positions of the histology sections were co-registered with images by cross-referencing the blocking diagram and photographs taken of the specimen during scanning, as illustrated in Fig. S2. Pathologists (BFD, $\mathrm{BL})$ determined tissue types present in the histology images. Engineers and pathologists worked together to confirm co-registration by cross-checking tissue features present in the histology images with similar features present in the OCT images, using the depth cross-section (B-scan) view of OCT to match the histology plane where needed, as described in (42).

To focus the histological analysis, and to facilitate the subsequent reader study, 3-D regions of interest (ROIs), $10 \times 10 \times 1 \mathrm{~mm}$, were selected from the wide-field $(45 \times 45 \times 1 \mathrm{~mm}) \mathrm{scans}$. At least one ROI was selected on every margin scanned in this study. ROls were then included in the reader study if they met the following criteria: good physical contact with the specimen; tissue not extensively damaged by thermal effects (due to cauterization during resection) as assessed by histopathology; and availability of a reliable histology match for the ROI. Considering that histology slices are typically taken every $\sim 3 \mathrm{~mm}, 1-3$ sections were available for a given ROI. ROls were designated as "positive" for cancer if the pathologist identified any tumor within $1 \mathrm{~mm}$ of the margin in the histology sections corresponding to the ROI, similar to designation of "positive" margins in other diagnostic accuracy studies in BCS specimens $(26,30)$.

\section{Reader study and statistical analysis}

The reader study was designed to have three main outcomes: diagnostic accuracy (sensitivity and specificity) of OCT, QME, and OCT plus QME. Seven readers (2 surgeons, 2 engineers, 1 medical sonographer, 1 pathology scientist and 1 medical resident), blinded to the histological results, participated in the study. First, readers were trained to read OCT images in a 1.5-hour session. They then completed a reading of all ROls (OCT alone, using criteria in Fig. 1A) within 10 days of training and had access to the training images during reading. Two weeks following the OCT training, readers were trained to read QME images in another 1.5-hour session. They then completed a reading of all ROls once more, this time viewing OCT and QME side-by-side, from which accuracy was calculated for QME (using criterion in Fig. 1B) and OCT plus QME (using criterion in Fig. 1C). To remove potential bias due to reader memory, the order and orientation of ROls were randomized between readings. In both readings, readers viewed the ROls $(10 \times 10 \times 1 \mathrm{~mm}$, presented as a stack of en face images displayed every $20 \mu \mathrm{m}$ up to $1 \mathrm{~mm}$ in depth) using the open source software ImageJ (v1.52a) (45), which allowed scrolling through depth to visualize the volume. Readers completed their evaluation using a custom-built interface in which they followed a set of criteria for the presence of cancer in each image type and selected "cancer" or "not cancer" as the endpoint (Fig. 1). 
Criteria for cancer in OCT images (Fig. 1A) were determined based on three main observations from prior OCT studies of breast tissues $(28,32,42,46,47)$ and pilot study data. First, it has been established that adipose tissue is easily distinguished from other tissues in breast based on its "honeycomb" structure in OCT. Therefore, readers were trained to first identify non-adipose features $>1 \mathrm{~mm}$ in diameter. If the ROI was made up of all adipose or adipose with only small $(<1$ $\mathrm{mm}$ ), isolated non-adipose features, it was considered "not cancer". Second, it was found in preliminary OCT studies on BCS specimens (42) and in this study that cancer near the margin tends to have continuity with depth, uninterrupted by regions of adipose tissue. Thus, if readers found a region of non-adipose tissue, they were to scroll through the 3D image, checking if the feature extended down into the specimen. Finally, a criterion was added to help distinguish benign stroma from regions of cancer. In benign stroma, a striated or banded pattern is observed in OCT, likely due to the organization of the underlying collagen. Cancer tends to disrupt this organization and result in heterogeneous patterns in $\operatorname{OCT}(31,48)$. One special case is mucinous carcinoma, (present in four recruited patients), in which tumor cells produce pools of a liquid substance known as mucin. Mucinous pools result in regions of low signal in OCT images, appearing similar to adipose tissue, but can often be identified by the outline of the pool, which has higher OCT signal. Thus, readers evaluated OCT images for the presence of these structures, even if at first it appeared that the tissue was adipose (bottom path, Fig 1A).

In QME images, readers were trained to look for areas of high elasticity, based on studies across spatial scales showing that cancer and its associated stroma are stiffer than benign tissues (36, $37,44)$. To avoid mistaking small, isolated patches of stiff stroma for cancer, the region of high elasticity had to cover at least $75 \%$ of a $1-\mathrm{mm}$ diameter circle (Fig. 1B). The user interface enabled readers to calculate this precisely by dragging their cursor to any location in the ROI. High elasticity was defined as $>26.3 \mathrm{kPa}$, based on analysis of prior and current study data and is denoted by a black line on the colorscale of all presented QME images. To assess OCT+QME in combination, readers were again directed to assess continuity with depth and ensure that the region was non-adipose tissue, in addition to the elasticity criterion (Fig. 1C).

Finally, the QME criterion, facilitated by its quantitative nature, was implemented into a preliminary automated algorithm. This involved thresholding the elasticity values in the QME images at every depth to generate binary images (with ones representing pixels with an elasticity $>26.3 \mathrm{kPa}$ ), then convolving these binary images with a $1 \mathrm{~mm}$ diameter circular kernel. The values at each pixel in the kernel were normalized such that the result of the convolution equaled the percentage of the circle covered by high elasticity. If, for a given ROI, the convolution produced a value $\geq 75 \%$ at any location in the ROI, that ROI was interpreted as containing cancer. As such, this algorithm was equivalent to the QME reader criteria shown in Fig. 1B. This algorithm was implemented on a standard desktop computer using MATLAB (Mathworks, R2016a), and took approximately one minute to read all ROls.

Following the readings, the sensitivity, specificity, positive predictive value (PPV), negative predictive value (NPV), and accuracy were calculated for each reader. 95\% confidence intervals were estimated using the score interval for a binomial case ('Wald' interval) (49). Aggregates were computed by taking the sum of the individual results of the seven readers (the totals of true positive, false positive, true negative, and false negative counts), and computing the sensitivity/specificity and confidence intervals on these summed totals. The performance of QME and OCT plus QME were compared against OCT alone using McNemar's test (50) for statistical significance. Inter-reader agreement was quantified using the Fleiss' Kappa metric (51). Detailed statistical calculations are provided in Supplementary Reader Statistics. 


\section{Results}

\section{OCT and QME images of malignant and benign breast tissues at the margin}

Intact BCS specimens from 71 patients were scanned for the reader study, without any damage to the tissue nor disruption to typical histopathological protocol. $174 \mathrm{ROls}$ from the 71 patients were selected and co-registered with histology. Of these, $154 \mathrm{ROls}$ were included in the reader study. Table 2 summarizes the tissue types and characteristics of the included ROls. Reasons for exclusion were extensive thermal damage, as determined by postoperative histology $(n=3)$; inconclusive registration with histology sections $(n=4)$; insufficient contact between the specimen, silicone layer, and the imaging window $(n=3)$; imaging artifacts (e.g., stray reflections or surgical clips precluded image interpretation, $n=4)$; insufficient elasticity data overlaid on nonadipose tissue $(n=2)$; a rare form of mucinous DCIS (insufficient data to build decision criteria, $n$ $=2$ ); and the region of tumor within the ROI having a size $<1 \mathrm{~mm}(n=2)$. Of the 154 ROls included in this study, 24 had cancer within $1 \mathrm{~mm}$ of the surface, corresponding to a prevalence of $15.6 \%$. Most were made up of a mix of tissue types, including adipose, stroma, and parenchymal tissues.

Figures 2-4 present ROls from the reader study, representative of the various tissue types encountered in the study. In each case, en face, $10 \times 10 \mathrm{~mm}$ OCT and QME images are displayed at a depth of 40-60 $\mu \mathrm{m}$ (entire 3-D volumes available in Supplementary Videos 1-6), along with the corresponding H\&E histology. The plane and orientation of the histology is indicated by the red dashed line in each set of images.

Figure 2 shows ROls containing benign breast tissues: adipose tissue (Fig. 2A) and a mix of adipose and stroma containing benign ducts (Fig. 2B). The OCT in Fig. 2A (Supp. Video 1) depicts the honeycomb structure typical of adipose tissue. Thin strands of connective tissue are present throughout this ROI, and QME shows that the elasticity in these regions tends to be low. Six of seven readers called this benign using OCT alone, compared to all readers using QME combined with OCT. A larger region of benign stroma is shown at the margin in Fig. 2B, and 3-D analysis reveals that this structure lacks continuity, with intermittent adipose tissue present with depth (Supp. Video 2), similar to the pattern in the histology image. However, using OCT alone, three readers incorrectly assessed this as cancer. In QME, this stroma exhibits mostly low elasticity. Very small pockets of high elasticity are seen at a few points throughout the stroma, but only features with more widespread elasticity were considered suspicious for cancer. All seven readers correctly called this benign using QME and QME plus OCT.

Figure 3 shows positive ROls from two BCS cases, each containing invasive ductal carcinoma on the margin. In Fig. 3A, the histology image reveals invasive ductal carcinoma grade III with high cellular density, surrounded by adipose tissue. Correspondingly, the OCT captured a region of non-adipose tissue with a nodular appearance (no indication of striated, organized collagen) that matches the location of tumor in the histology image. In QME, this region shows elevated elasticity, due to the much higher cellular density in the tumor compared to the surrounding adipose tissue. This detected region of tumor is $\sim 2 \mathrm{~mm}$ across, making up $<0.2 \%$ of the total surface area of this particular margin, demonstrating the high resolution of the technique. All seven readers evaluated this ROI correctly using OCT, QME, and the combination. The histology image in Fig. 3B shows invasive ductal carcinoma grade III surrounded by a mix of stroma and adipose tissue. A mix of adipose and non-adipose tissue is also present in the OCT image, and QME reveals a portion of the non-adipose to have high elasticity. 3-D analysis of the images (Supp. Video 4) shows that the region with high elasticity is also continuous with depth (i.e., adipose tissue is not revealed underneath). Based on OCT alone, one of the seven readers called this as a false negative, mistaking it for benign, whereas all seven readers evaluated this as cancer using QME alone or alongside OCT. 
Figure 4 shows ROls containing two malignant tissue types: DCIS (Fig. 4A) and mucinous carcinoma (Fig. 4B), which were designated "impalpable" pre-operatively and required hookwire guidance for lesion excision. Despite being impalpable on a macro-scale, changes in mechanical properties on a micro-scale are detectable by QME for these tissue types (43). Figure 4A shows an example of DCIS within $0.15 \mathrm{~mm}$ of the margin; the histology image shows the involved duct surrounded by dense, benign stroma. The OCT shows no apparent contrast between this duct and the surrounding stroma, but the QME highlights high elasticity corresponding to the region of DCIS. This elevated elasticity corresponds to the dense, proliferating cells within the duct, as well as a fibrotic stromal response (52) immediately surrounding the duct. Throughout the stroma in the rest of the ROI, there are small, localized areas of elevated elasticity, but most do not meet the size criterion for cancer defined in this study. Six of seven readers evaluated this correctly using OCT alone, as the structure shows continuity with depth (Supp. Video 5), and all readers evaluated it correctly using QME. In Fig. 4B (Supp. Video 6), a region of benign stroma and adipose tissue neighbors a region of mucinous carcinoma on the margin. In OCT, the region corresponding to the mucinous tumor is difficult to distinguish from the surrounding adipose tissue, and one reader designated this as benign. However, in QME the tumor exhibits elevated elasticity, and all readers correctly classified this $\mathrm{ROI}$ as cancerous when QME was available.

\section{Blinded reader study results in higher accuracy using QME over OCT alone}

Table 3 and Fig. S3 summarize the results of the reader study. Readers completed evaluation of the 154 OCT plus QME ROls in an average of 4 hours, or $\sim 90$ seconds per ROI. Inter-reader agreement was nearly perfect for QME and OCT plus QME, and moderate for OCT alone, according to a standard interpretation of the Fleiss' Kappa index for interrater agreement. Reported ranges for sensitivity and specificity in Table 3 indicate 95\% confidence intervals. Based on the aggregate results, the sensitivity, specificity, and accuracy of OCT for detecting cancer within $1 \mathrm{~mm}$ of the margin were $69.0 \% 79.0 \%$, and $77.5 \%$, respectively. Using QME, sensitivity, specificity, and accuracy were $92.9 \%, 96.4 \%$, and 95.8\%, respectively. Using combined OCT and QME criteria, sensitivity, specificity, and accuracy were $80.4 \%, 99.5 \%$, and $96.5 \%$, respectively. Sensitivity was significantly $(p<0.05)$ improved using QME over OCT alone for 6 of the 7 readers, and specificity was significantly improved using QME over OCT alone for all 7 readers (see Supplementary Reader Statistics for calculations).

Also reported in Table 3, the automated reader for QME resulted in 100.0\%, 97.7\%, and 98.1\% sensitivity, specificity, and accuracy, respectively.

\section{Discussion}

This study is the first to determine the accuracy of QME, or any variant of optical elastography, for assessment of tumor margins in specimens excised during breast-conserving surgery. Prior to this study, recent investigations had demonstrated that QME has the potential to delineate tumor in breast tissue based on elevated elasticity on a micro-scale $(43,44)$. In these studies, as in this one, the contrast between tumor and healthy tissue based on changes in elasticity was shown to be complementary to the structural contrast provided by the underlying modality, OCT. However, prior studies had not investigated the ability of QME to identify positive margins in specimens from breast-conserving surgery. This study has demonstrated high accuracy of QME $(96 \%)$ compared to OCT (78\%) for detecting cancer within $1 \mathrm{~mm}$ of the margin in BCS specimens. The measurements here, on intact BCS specimens immediately following surgery, are also of high clinical relevance, compared to prior OCT studies that relied primarily on dissected mastectomy specimens for testing novel margin assessment techniques $(27,28,46)$. 
The QME results here indicate that tissue elasticity is an accurate predictor of malignancy. This trend was consistent across multiple tumor types in this study (e.g., solid and mucinous invasive carcinomas, and DCIS), although this must be confirmed with larger sample sizes of each tumor type. Future data collection will also continue to elucidate the sources of elevated elasticity in each tumor type, e.g., deposition of stiff desmoplastic stroma, changes in collagen alignment, or interstitial fluid pressure. Better understanding of these mechanisms may foster even greater precision in the use of QME to detect various tumor types intraoperatively. This goal may be facilitated by implementing ultrahigh resolution QME to achieve cellular-scale elasticity resolution $(53,54)$.

Perhaps counter-intuitively, whilst combining OCT and QME improved overall accuracy compared to OCT alone, the sensitivity using the high elasticity criterion (93\%) was greater than that using QME and OCT criteria together (80\%). This may be due to variability in reader interpretation of the qualitative OCT criteria (interpretation of structures) versus little variability in interpretation of the quantitative QME criterion (elasticity threshold). In particular, analysis of reader notes revealed variability in whether readers considered features as "continuous" with depth based on OCT (Fig. 1A), which was requisite in this study for an ROI to be considered positive. (The engineers who participated as readers had prior experience in reading OCT and elastography images, which may have contributed to their slightly superior reading performance.) On the other hand, specificity of OCT and QME together ( 100\%) was greater than that of QME $(96 \%)$ or OCT $(79 \%)$ alone, indicating that the combination of information reduces false positives (e.g., mistaking benign, fibrous stroma for cancer). To improve intraoperative margin assessment and ultimately reduce the need for costly repeat surgeries, high sensitivity is essential to ensuring that less cancer is missed (i.e., minimizing false negatives). High specificity, on the other hand, ensures that less benign tissue is unnecessarily removed, and, while important to avoid over-treatment of the cancer, could be considered secondary to the need for high sensitivity in margin assessment. QME showed the best overall performance on both sides, but future studies will need to analyze the amount of repeat surgeries avoided versus extra volumes of tissue removed.

While the accuracy of OCT reported here is similar to that reported in another study in BCS specimens (30), further refinement and quantification of OCT criteria may potentially increase accuracy in future studies. For instance, quantification of the rate of OCT signal attenuation with depth has shown potential for distinguishing benign and malignant regions $(28,32)$, though it is not clear the extent to which this will improve accuracy. Our preliminary automation of QME reading could also be extended to incorporate both QME and OCT-based information into an algorithm utilizing neural networks.

While the spatial resolution of OCT and QME images is on the order of micrometers (Fig. S4), which is comparable or superior to many existing techniques, readers were directed to ignore features smaller than $\sim 1 \mathrm{~mm}$. This set an effective "diagnostic resolution" of $\sim 1 \mathrm{~mm}$, and further study is needed to assess the accuracy of OCT and QME for smaller features, such as very early stage DCIS. However, at a more advanced stage, DCIS tends to result in dilation of ducts, and even small ducts can result in a fibrous stromal response in the surrounding tissue (52); thus, QME is expected to be sensitive to the associated mechanical changes caused by these features. The resolution of the technique may also improve in the future using the inverse method to solve for elasticity $(55,56)$, rather than rely on assumptions of stress uniformity with depth, as in our current method; however, computation times currently prohibit this from being implemented in clinically feasible timeframes.

Some ROls containing tissue that was thermally damaged by cauterization during surgery were excluded from this study, as it is difficult to achieve accurate histology reading in regions with 
extensive thermal damage. In addition, in some instances, thermal damage can result in high elasticity of tissues that are benign. As thermal damage may be difficult to completely avoid in clinical practice, future studies could mitigate its presence in the images by using combined contrast from QME and OCT, including investigation of textural analysis, as well as colorimetric data from photographs simultaneously taken of the specimen (Fig. S1), which often show black marks on the tissue surface that may correspond to thermal damage.

Two margins per specimen were imaged in this study, at a rate of $\sim 9$ minutes per margin. In some specimens, post-operative histology revealed cancer within $1 \mathrm{~mm}$ on margins that were not scanned, leading to a discrepancy in the overall prevalence of close margins reported in Table 1 (45\%) and the prevalence of ROls containing cancer (15.6\%). To facilitate rapid intraoperative QME of all radial margins, which is desirable in the clinical scenario, higher acquisition speed has been achieved using a novel approach (57), and may be further increased by implementation of high-speed OCT systems, an order of magnitude faster than that in this study (58). In addition, reader evaluation of the images took 90 seconds on average, which is conducive to clinical timeframes. Finally, a handheld probe is in development to allow QME assessment of tissues directly within the surgical cavity and to facilitate implementation into the clinical workflow (59).

In summary, QME, an emerging OCT elastography technique that probes tissue elasticity on the micro-scale, has demonstrated detection of close and positive margins in freshly excised specimens in BCS with $93 \%$ sensitivity and $96 \%$ specificity. The use of intrinsic tissue contrast without need for exogenous dyes and the optimal trade-off in speed, field of view, and resolution provided by QME make it a promising candidate for improving intraoperative guidance of BCS. More broadly, QME may be applicable in a range of surgical or preoperative biopsy guidance applications, particularly in cancers that are known to exhibit altered mechanical properties.

\section{Acknowledgements}

We gratefully acknowledge Dr. Max Bulsara from University of Notre Dame Australia for discussion of biostatistics.

\section{References}

1. Landercasper J, Attai D, Atisha D, Beitsch P, Bosserman L, Boughey J, Carter J, Edge S, Feldman S, Froman J. Toolbox to reduce lumpectomy reoperations and improve cosmetic outcome in breast cancer patients: the American Society of Breast Surgeons Consensus Conference. Annals of Surgical Oncology. 2015;22(10):3174-83.

2. Landercasper J, Whitacre E, Degnim AC, Al-Hamadani M. Reasons for re-excision after lumpectomy for breast cancer: insight from the American Society of Breast Surgeons Mastery SM database. Annals of Surgical Oncology. 2014;21(10):3185-91.

3. McCahill LE, Single RM, Bowles EJA, Feigelson HS, James TA, Barney T, Engel JM, Onitilo AA. Variability in reexcision following breast conservation surgery. JAMA: The Journal of the American Medical Association. 2012;307(5):467-75.

4. Kimball CC, Nichols Cl, Vose JG. The Payer and Patient Cost Burden of Open Breast Conserving Procedures Following Percutaneous Breast Biopsy. Breast Cancer: Basic Clinical Research. 2018. doi: 1178223418777766. 
5. Metcalfe LN, Zysk AM, Yemul KS, Jacobs LK, Oker EE, Underwood HR, Thompson AM. Beyond the margins - economic costs and complications associated with repeated breastconserving surgeries. JAMA Surgery. 2017;152(11):1084-6.

6. Esbona K, Li Z, Wilke LG. Intraoperative imprint cytology and frozen section pathology for margin assessment in breast conservation surgery: a systematic review. Annals of Surgical Oncology. 2012;19(10):3236-45.

7. Decker MR, Trentham-Dietz A, Loconte NK, Neuman HB, Smith MA, Punglia RS, Greenberg CC, Wilke LG. The role of intraoperative pathologic assessment in the surgical management of ductal carcinoma in situ. Annals of Surgical Oncology. 2016;23(9):2788-94.

8. Harness JK, Giuliano AE, Pockaj BA, Downs-Kelly E. Margins: a status report from the Annual Meeting of the American Society of Breast Surgeons. Annals of Surgical Oncology. 2014;21(10):3192-7.

9. Miller CL, Coopey SB, Rafferty E, Gadd M, Smith BL, Specht MC. Comparison of intraoperative specimen mammography to standard specimen mammography for excision of non-palpable breast lesions: a randomized trial. Breast Cancer Research and Treatment. 2016;155(3):513-9.

10. Rhee D, Pockaj B, Wasif N, Stucky C-C, Pizzitola V, Giurescu M, Patel B, McCarthy J, Gray R. Operative outcomes of conventional specimen radiography versus in-operating room specimen radiography in radioactive seed-localized segmental mastectomies. The American Journal of Surgery. 2018;215(1):151-4.

11. Britton P, Sonoda L, Yamamoto A, Koo B, Soh E, Goud A. Breast surgical specimen radiographs: how reliable are they? European Journal of Radiology. 2011;79(2):245-9.

12. Layfield DM, May DJ, Cutress RI, Richardson C, Agrawal A, Wise M, Yiangou C. The effect of introducing an in-theatre intra-operative specimen radiography (IOSR) system on the management of palpable breast cancer within a single unit. The Breast. 2012;21(4):459-63. doi: 10.1016/j.breast.2011.10.010.

13. Krekel NM, Haloua MH, Cardozo AML, de Wit RH, Bosch AM, de Widt-Levert LM, Muller S, van der Veen $\mathrm{H}$, Bergers $\mathrm{E}$, de Klerk ESdL. Intraoperative ultrasound guidance for palpable breast cancer excision (COBALT trial): a multicentre, randomised controlled trial. The Lancet Oncology. 2013;14(1):48-54.

14. Ramos M, Díaz JC, Ramos T, Ruano R, Aparicio M, Sancho M, González-Orús JM. Ultrasoundguided excision combined with intraoperative assessment of gross macroscopic margins decreases the rate of reoperations for non-palpable invasive breast cancer. The Breast. 2013;22(4):520-4.

15. Ahmed M, Douek M. Intra-operative ultrasound versus wire-guided localization in the surgical management of non-palpable breast cancers: systematic review and metaanalysis. Breast Cancer Research and Treatment. 2013;140(3):435-46.

16. Olsha O, Shemesh D, Carmon M, Sibirsky O, Dalo RA, Rivkin L, Ashkenazi I. Resection margins in ultrasound-guided breast-conserving surgery. Annals of Surgical Oncology. 2011;18(2):447-52.

17. Pappo I, Spector R, Schindel A, Morgenstern S, Sandbank J, Leider LT, Schneebaum S, Lelcuk $S$, Karni T. Diagnostic performance of a novel device for real-time margin assessment in lumpectomy specimens. Journal of Surgical Research. 2010;160(2):277-81. 
18. Schnabel F, Boolbol SK, Gittleman M, Karni T, Tafra L, Feldman S, Police A, Friedman NB, Karlan S, Holmes D. A randomized prospective study of lumpectomy margin assessment with use of MarginProbe in patients with nonpalpable breast malignancies. Annals of Surgical Oncology. 2014;21(5):1589-95.

19. Lamberts LE, Koch M, de Jong JS, Adams AL, Glatz J, Kranendonk ME, van Scheltinga AGT, Jansen L, de Vries J, Lub-de Hooge MN. Tumor-specific uptake of fluorescent bevacizumabIRDye800CW microdosing in patients with primary breast cancer: a phase i feasibility study. J Clinical Cancer Research. 2017;23(11):2730-41.

20. Tummers QR, Verbeek FP, Schaafsma BE, Boonstra MC, van der Vorst JR, Liefers G-J, van de Velde CJ, Frangioni JV, Vahrmeijer AL. Real-time intraoperative detection of breast cancer using near-infrared fluorescence imaging and methylene blue. European Journal of Surgical Oncology. 2014;40(7):850-8.

21. Unkart JT, Chen SL, Wapnir IL, González JE, Harootunian A, Wallace AM. Intraoperative tumor detection using a ratiometric activatable fluorescent peptide: a first-in-human phase 1 study. Annals of Surgical Oncology. 2017;24(11):3167-73.

22. Whitley MJ, Cardona DM, Lazarides AL, Spasojevic I, Ferrer JM, Cahill J, Lee C-L, Snuderl M, Blazer DG, Hwang ES. A mouse-human phase 1 co-clinical trial of a protease-activated fluorescent probe for imaging cancer. Science Translational Medicine. 2016;8(320):320ra4ra4.

23. Wang YW, Reder NP, Kang S, Glaser AK, Yang Q, Wall MA, Javid SH, Dintzis SM, Liu JTC. Raman-Encoded Molecular Imaging with Topically Applied SERS Nanoparticles for Intraoperative Guidance of Lumpectomy. Cancer Research. 2017;77(16):4506-16.

24. Brown JQ, Bydlon TM, Kennedy SA, Caldwell ML, Gallagher JE, Junker M, Wilke LG, Barry WT, Geradts J, Ramanujam N. Optical Spectral Surveillance of Breast Tissue Landscapes for Detection of Residual Disease in Breast Tumor Margins. PLOS ONE. 2013;8(7):e69906.

25. Kho E, de Boer LL, Van de Vijver K, van Duijnhoven F, Vrancken Peeters M-JTFD, Sterenborg HJCM, Ruers TJ. Hyperspectral imaging for resection margin assessment during cancer surgery. Clinical Cancer Research. 2019: doi: 10.1158/1078-0432.CCR-18-2089.

26. Shipp DW, Rakha EA, Koloydenko AA, Macmillan RD, Ellis IO, Notingher I. Intra-operative spectroscopic assessment of surgical margins during breast conserving surgery. Breast Cancer Res. 2018;20(1):69.

27. Erickson-Bhatt SJ, Nolan RM, Shemonski ND, Adie SG, Putney J, Darga D, McCormick DT, Cittadine AJ, Zysk AM, Marjanovic M, Chaney EJ, Monroy GL, South FA, Cradock KA, Liu ZG, Sundaram M, Ray PS, Boppart SA. Real-time Imaging of the Resection Bed Using a Handheld Probe to Reduce Incidence of Microscopic Positive Margins in Cancer Surgery. Cancer Research. 2015;75(18):3706..

28. Ha R, Friedlander LC, Hibshoosh H, Hendon C, Feldman S, Ahn S, Schmidt H, Akens MK, Fitzmaurice $M$, Wilson $B C$. Optical coherence tomography: A novel imaging method for post-lumpectomy breast margin assessment-A multi-reader study. Academic Radiology. 2018;25(3):279-87.

29. Yao X, Gan Y, Chang E, Hibshoosh H, Feldman S, Hendon C. Visualization and tissue classification of human breast cancer images using ultrahigh-resolution OCT. Lasers in Surgery and Medicine. 2017;49(3):258-69. 
30. Zysk AM, Chen K, Gabrielson E, Tafra L, May Gonzalez EA, Canner JK, Schneider EB, Cittadine AJ, Scott Carney P, Boppart SA, Tsuchiya K, Sawyer K, Jacobs LK. Intraoperative Assessment of Final Margins with a Handheld Optical Imaging Probe During Breast-Conserving Surgery May Reduce the Reoperation Rate: Results of a Multicenter Study. Annals of Surgical Oncology. 2015;22(10):3356-62.

31. Kennedy BF, McLaughlin RA, Kennedy KM, Chin L, Wijesinghe P, Curatolo A, Tien A, Ronald M, Latham B, Saunders CM, Sampson DD. Investigation of optical coherence microelastography as a method to visualize cancers in human breast tissue. Cancer Research. 2015.75(16)3236-3245.

32. Scolaro L, McLaughlin RA, Kennedy BF, Saunders CM, Sampson DD. A review of optical coherence tomography in breast cancer. Photonics \& Lasers in Medicine. 2014;3(3):22540.

33. Zhou C, Cohen DW, Wang Y, Lee HC, Mondelblatt AE, Tsai TH, Aguirre AD, Fujimoto JG, Connolly JL. Integrated Optical Coherence Tomography and Microscopy for Ex Vivo Multiscale Evaluation of Human Breast Tissues. Cancer Research. 2010;70(24):10071.

34. Maloney BW, McClatchy DM, Pogue BW, Paulsen KD, Wells WA, Barth RJ. Review of methods for intraoperative margin detection for breast conserving surgery. Journal of Biomedical Optics. 2018;23(10):1-19, .

35. McEvoy MP, Landercasper J, Naik HR, Feldman S. Update of the American Society of Breast Surgeons Toolbox to address the lumpectomy reoperation epidemic. Gland Surgery. 2018;7(6):536.

36. Krouskop TA, Wheeler TM, Kallel F, Garra BS, Hall T. Elastic moduli of breast and prostate tissues under compression. Ultrasonic Imaging. 1998;20(4):260-74.

37. Plodinec M, Loparic M, Monnier CA, Obermann EC, Zanetti-Dallenbach R, Oertle $P$, Hyotyla JT, Aebi U, Bentires-Alj M, Lim RY. The nanomechanical signature of breast cancer. Nature Nanotechnology. 2012;7(11):757-65.

38. Wojcinski S, Farrokh A, Weber S, Thomas A, Fischer T, Slowinski T, Schmidt W, Degenhardt F. Multicenter study of ultrasound real-time tissue elastography in 779 cases for the assessment of breast lesions: improved diagnostic performance by combining the $\mathrm{BI}$ RADS $\neg \mathbb{E}$-US classification system with sonoelastography. Ultraschall in der Medizin. 2010;31(05):484-91.

39. Dua SM, Gray RJ, Keshtgar M. Strategies for localisation of impalpable breast lesions. The Breast. 2011;20(3):246-53. doi: https://doi.org/10.1016/i.breast.2011.01.007.

40. Xu H, Varghese T, Jiang J, Zagzebski JA. In vivo classification of breast masses using features derived from axial-strain and axial-shear images. Ultrasonic Imaging. 2012;4:222-36.

41. Kennedy BF, Wijesinghe $P$, Sampson DD. The emergence of optical elastography in biomedicine. Nature Photonics. 2017;11:215. doi: 10.1038/nphoton.2017.6.

42. Allen WM, Foo KY, Zilkens R, Kennedy KM, Fang Q, Chin L, Dessauvagie BF, Latham B, Saunders CM, Kennedy BF. Clinical feasibility of optical coherence micro-elastography for imaging tumor margins in breast-conserving surgery. Biomedical Optics Express. 2018;9(12):6331-49.

43. Kennedy KM, Chin L, McLaughlin RA, Latham B, Saunders CM, Sampson DD, Kennedy BF. Quantitative micro-elastography: imaging tissue elasticity using compression optical coherence elastography. Scientific Reports. 2015;5:15538. 
44. Allen WM, Kennedy KM, Fang Q, Chin L, Curatolo A, Watts L, Zilkens R, Chin SL, Dessauvagie BF, Latham B, Saunders CM, Kennedy BF. Wide-field quantitative micro-elastography of human breast tissue. Biomedical Optics Express. 2018;9(3):1082-96..

45. Schneider CA, Rasband WS, Eliceiri KW. NIH Image to ImageJ: 25 years of image analysis. Nature Methods. 2012;9:671. doi: 10.1038/nmeth.2089.

46. Assayag O, Antoine M, Sigal-Zafrani B, Riben M, Harms F, Burcheri A, Grieve K, Dalimier E, Le Conte dPB, Boccara C. Large Field, High Resolution Full-Field Optical Coherence Tomography: A Pre-clinical Study of Human Breast Tissue and Cancer Assessment. Technology in Cancer Research \& Treatment. 2013.

47. Nguyen FT, Zysk AM, Chaney EJ, Kotynek JG, Oliphant UJ, Bellafiore FJ, Rowland KM, Johnson PA, Boppart SA. Intraoperative evaluation of breast tumor margins with optical coherence tomography. Cancer research. 2009;69(22):8790.

48. Yemul KS, Zysk AM, Richardson AL, Tangella KV, Jacobs LK. Interpretation of Optical Coherence Tomography Images for Breast Tissue Assessment. Surgical Innovation. 2019;26(1):50-6. doi: 10.1177/1553350618803245. PubMed PMID: 30295149.

49. Brown LD, Cai TT, DasGupta A. Interval Estimation for a Binomial Proportion. Statistical Science. 2001;16(2):101-17.

50. Kim S, Lee W. Does McNemar's test compare the sensitivities and specificities of two diagnostic tests? Statistical methods in medical research. 2017;26(1):142-54.

51. Fleiss JL. Measuring nominal scale agreement among many raters. Psychological bulletin. 1971;76(5):378.

52. Sharma M, Beck AH, Webster JA, Espinosa I, Montgomery K, Varma S, van de Rijn M, Jensen KC, West RB. Analysis of stromal signatures in the tumor microenvironment of ductal carcinoma in situ. Breast Cancer Research and Treatment. 2010;123(2):397-404.

53. Curatolo A, Villiger M, Lorenser D, Wijesinghe $P$, Fritz A, Kennedy BF, Sampson DD. Ultrahigh resolution optical coherence elastography. Optics Letters. 2016; 41(1):21-24.

54. Wijesinghe P, Johansen NJ, Curatolo A, Sampson DD, Ganss R, and Kennedy BF. Ultrahighresolution optical coherence elastography images cellular-scale stiffness of mouse aorta. Biophysical Journal. 2017; 113(11):2540-2551.

55. Dong L, Wijesinghe P, Dantuono J, Sampson D, Munro P, Kennedy B, Oberai A. Quantitative Compression Optical Coherence Elastography as an Inverse Elasticity Problem. IEEE Journal of Selected Topics in Quantum Electronics. 2016;22(3).

56. Dong L, Wijesinghe P, Sampson DD, Kennedy BF, Munro PR, Oberai AA. Volumetric quantitative optical coherence elastography with an iterative inversion method. Biomedical Optics Express. 2019;10(2):384-98.

57. Fang Q, Frewer L, Wijesinghe $P$, Allen WM, Chin L, Hamzah J, Sampson DD, Curatolo A, Kennedy BF. Depth-encoded optical coherence elastography for simultaneous volumetric imaging of two tissue faces. Optics Letters. 2017;42(7):1233-6.

58. Klein T, Huber R. High-speed OCT light sources and systems [Invited]. Biomedical Optics Express. 2017;8(2):828-59.

59. Fang Q, Krajancich B, Chin L, Zilkens R, Curatolo A, Frewer L, Anstie JD, Wijesinghe P, Hall C, Dessauvagie BF, Latham B, Saunders CM, Kennedy BF. Handheld probe for quantitative micro-elastography. Biomedical Optics Express. 2019;10(8):4034-49. 
Table 1. BCS patient and clinical specimen characteristics.

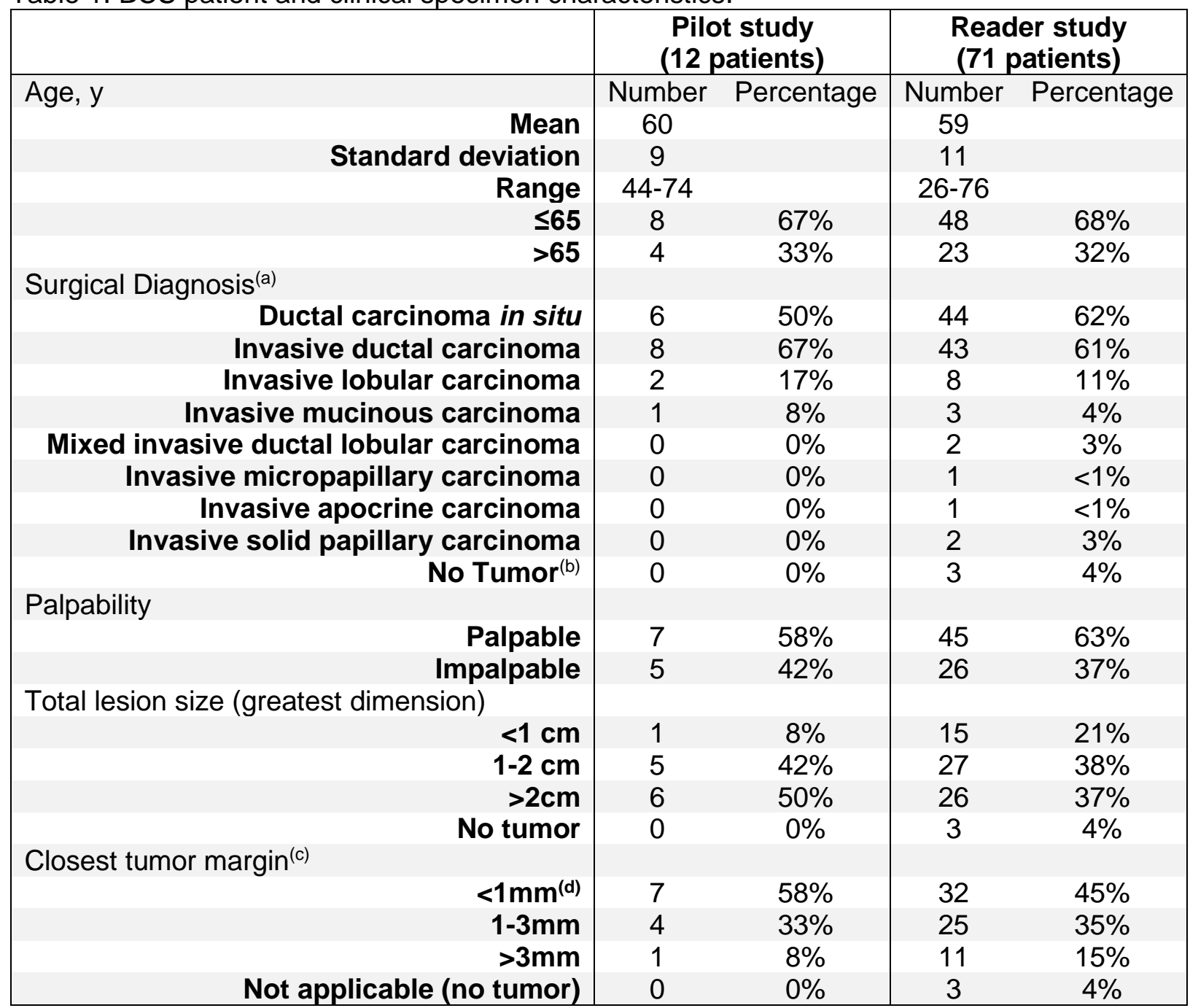

(a) Multiple tumor types may occur in a given patient, such that diagnosis percentages add to $>100 \%$

(b) Tumor was excised in original core biopsy

(c) Includes deep and superficial margins in addition to radial margins

(d) $13(18 \%)$ of BCS specimens in the reader study had positive margins (i.e. tumor on ink) 
Table 2. Summary of regions of interest included in study.

\begin{tabular}{|rcc|} 
ROI selection & Number & \\
ROls co-registered with histology & 174 & \\
ROls included in reader study & 154 & \\
Tissue types in included ROls & & Percentage (/154) \\
Cancer within 1 mm (positive ROI) & $\mathbf{2 4}$ & $\mathbf{1 5 . 6 \%}$ \\
Invasive (solid) & 14 & $9.1 \%$ \\
Invasive (mucinous) & 3 & $1.9 \%$ \\
DCIS & 7 & $4.5 \%$ \\
Clear within 1 mm (negative ROI) & $\mathbf{1 3 0}$ & $\mathbf{8 4 . 4 \%}$ \\
Predominantly adipose & 38 & $24.7 \%$ \\
Adipose with dense stroma & 37 & $24.0 \%$ \\
Adipose with ducts/vessels & 24 & $15.6 \%$ \\
Adipose with strands of connective tissue & 19 & $12.3 \%$ \\
Dense stroma & 12 & $7.8 \%$ \\
\hline
\end{tabular}


Table 3. Reader study results.

\begin{tabular}{|c|c|c|c|c|c|c|c|c|c|c|c|c|c|c|c|}
\hline \multirow[b]{2}{*}{ Reader } & \multicolumn{5}{|l|}{ OCT } & \multicolumn{5}{|l|}{ QME } & \multicolumn{5}{|l|}{$\mathrm{OCT}+\mathrm{QME}$} \\
\hline & Sensitivity & Specificity & PPV & NPV & Acc & Sensitivity & Specificity & PPV & NPV & Acc & Sensitivity & Specificity & PPV & NPV & Acc \\
\hline Eng 1 & $62.5 \pm 19.4 \%$ & $85.4 \pm 6.1 \%$ & $44 \%$ & $93 \%$ & $82 \%$ & $100.0 \pm 0.0 \%$ & $97.7 \pm 2.6 \%$ & $89 \%$ & $100 \%$ & $98 \%$ & $87.5 \pm 13.2 \%$ & $100 \pm 0.0 \%$ & $100 \%$ & $98 \%$ & $98 \%$ \\
\hline Eng 2 & $70.8 \pm 18.2 \%$ & $86.9 \pm 5.8 \%$ & $50 \%$ & $94 \%$ & $84 \%$ & $100.0 \pm 0.0 \%$ & $97.7 \pm 2.6 \%$ & $89 \%$ & $100 \%$ & $98 \%$ & $87.5 \pm 13.2 \%$ & $100 \pm 0.0 \%$ & $100 \%$ & $98 \%$ & $98 \%$ \\
\hline Surg 1 & $62.5 \pm 19.4 \%$ & $74.6 \pm 7.5 \%$ & $31 \%$ & $92 \%$ & $73 \%$ & $75.0 \pm 17.3 \%$ & $88.5 \pm 5.5 \%$ & $55 \%$ & $95 \%$ & $86 \%$ & $70.8 \pm 18.2 \%$ & $96.9 \pm 3.0 \%$ & $81 \%$ & $95 \%$ & $93 \%$ \\
\hline Surg 2 & $66.7 \pm 18.9 \%$ & $72.3 \pm 7.7 \%$ & $31 \%$ & $92 \%$ & $71 \%$ & $95.8 \pm 8.0 \%$ & $97.7 \pm 2.6 \%$ & $89 \%$ & $99 \%$ & $97 \%$ & $95.8 \pm 8.0 \%$ & $100 \pm 0.0 \%$ & $100 \%$ & $99 \%$ & $99 \%$ \\
\hline Path & $66.7 \pm 18.9 \%$ & $83.8 \pm 6.3 \%$ & $43 \%$ & $93 \%$ & $81 \%$ & $95.8 \pm 8.0 \%$ & $97.7 \pm 2.6 \%$ & $89 \%$ & $99 \%$ & $97 \%$ & $70.8 \pm 18.2 \%$ & $100 \pm 0.0 \%$ & $100 \%$ & $95 \%$ & $96 \%$ \\
\hline Res & $79.2 \pm 16.2 \%$ & $74.6 \pm 7.5 \%$ & $37 \%$ & $95 \%$ & $75 \%$ & $87.5 \pm 13.2 \%$ & $97.7 \pm 2.6 \%$ & $88 \%$ & $98 \%$ & $96 \%$ & $66.7 \pm 18.9 \%$ & $99.2 \pm 1.5 \%$ & $94 \%$ & $94 \%$ & $94 \%$ \\
\hline Sonog & $75.0 \pm 17.3 \%$ & $75.4 \pm 7.4 \%$ & $36 \%$ & $94 \%$ & $75 \%$ & $95.8 \pm 8.0 \%$ & $97.7 \pm 2.6 \%$ & $89 \%$ & $99 \%$ & $97 \%$ & $83.3 \pm 14.9 \%$ & $100 \pm 0.0 \%$ & $100 \%$ & $97 \%$ & $97 \%$ \\
\hline Agg & $69.0 \pm 7.0 \%$ & $79.0 \pm 2.6 \%$ & $38 \%$ & $93 \%$ & $78 \%$ & $92.9 \pm 3.9 \%$ & $96.4 \pm 1.2 \%$ & $83 \%$ & $99 \%$ & $96 \%$ & $80.4 \pm 6.0 \%$ & $99.5 \pm 0.5 \%$ & $96 \%$ & $97 \%$ & $97 \%$ \\
\hline
\end{tabular}

\begin{tabular}{|r|lllll|llllllllllllll}
\hline $\begin{array}{r}\text { Automated } \\
\text { reader }\end{array}$ & - & & - & - & - & - & - & $100.0 \pm 0.0 \%$ & $97.7 \pm 2.6 \%$ & $89 \%$ & $100 \%$ & $98 \%$ & - & - & -
\end{tabular}

Eng: Engineer; Surg: Surgeon; Path: Pathology scientist; Res: Medical resident; Sonog: Sonographer; PPV: Positive predictive value; NPV: Negative predictive value; Acc: Accuracy; Agg: Aggregate 

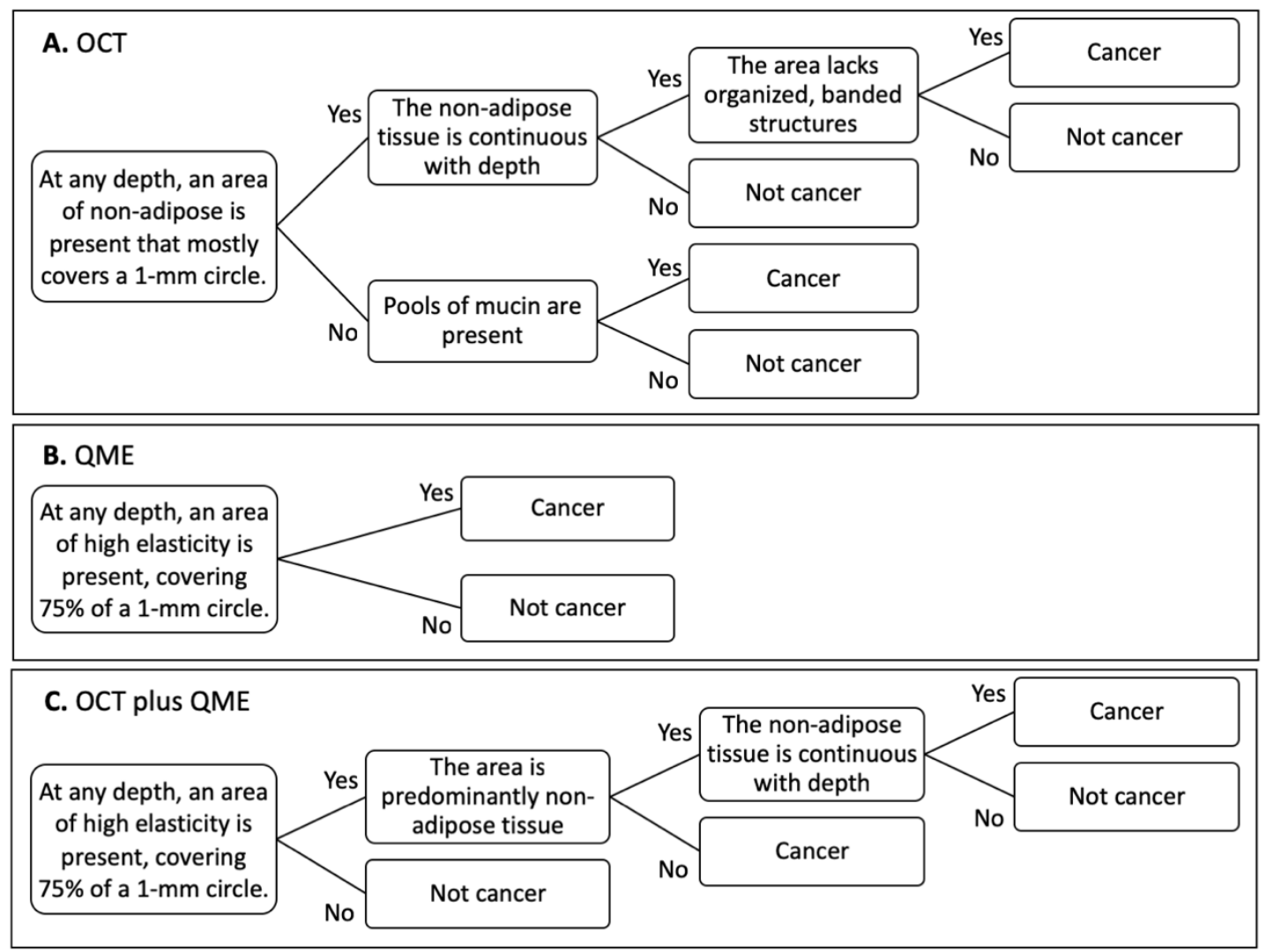

Figure 1. Reader criteria for determining presence of cancer. Criteria for cancer using (A) OCT images, (B) elasticity in QME images, (C) OCT and QME combined.

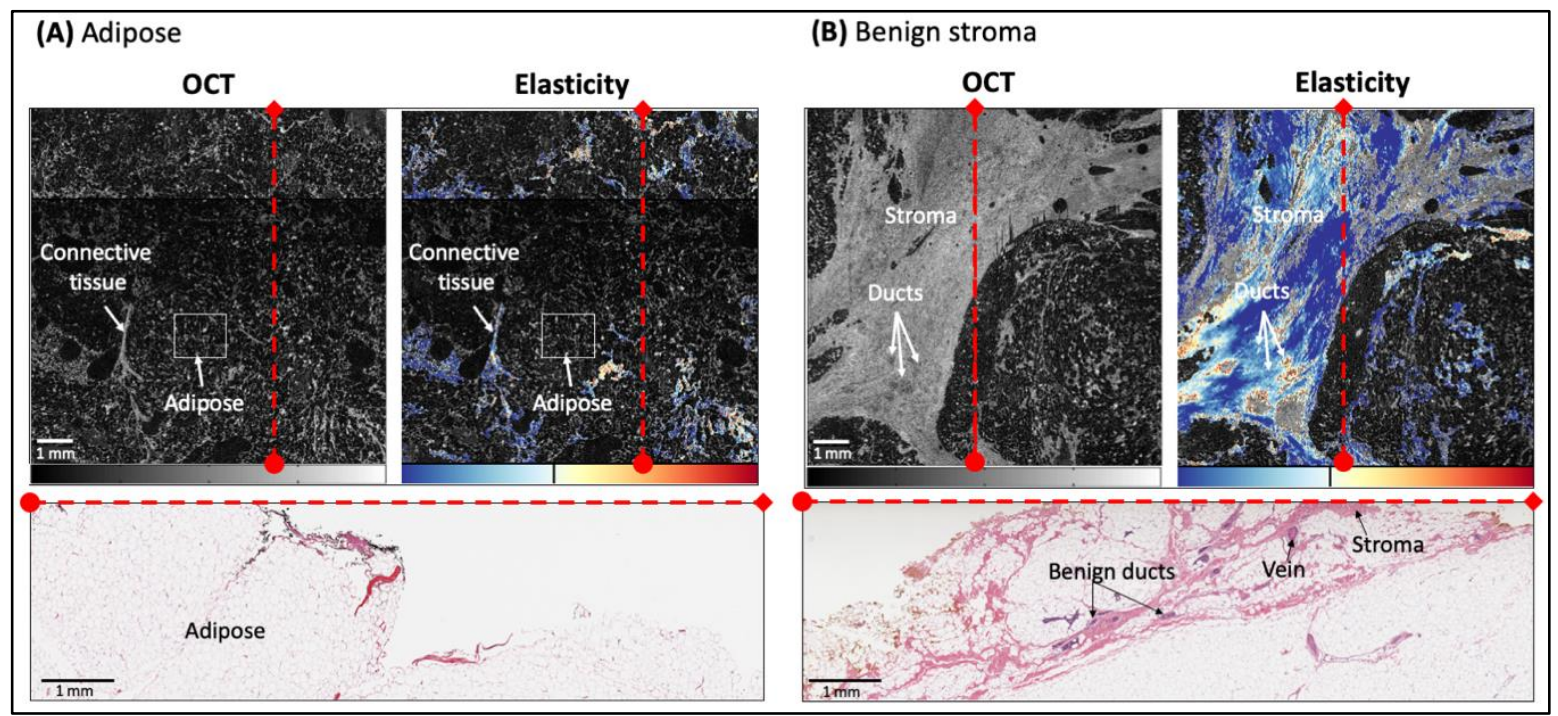

Figure 2. Examples images of benign breast tissues. OCT, QME, and H\&E histology of (A) adipose tissue with thin strands of stroma and $(B)$ a large region of benign stroma and adipose tissues. Colorbars: OCT 0-40 dB; Elasticity 3.63-363 kPa. 


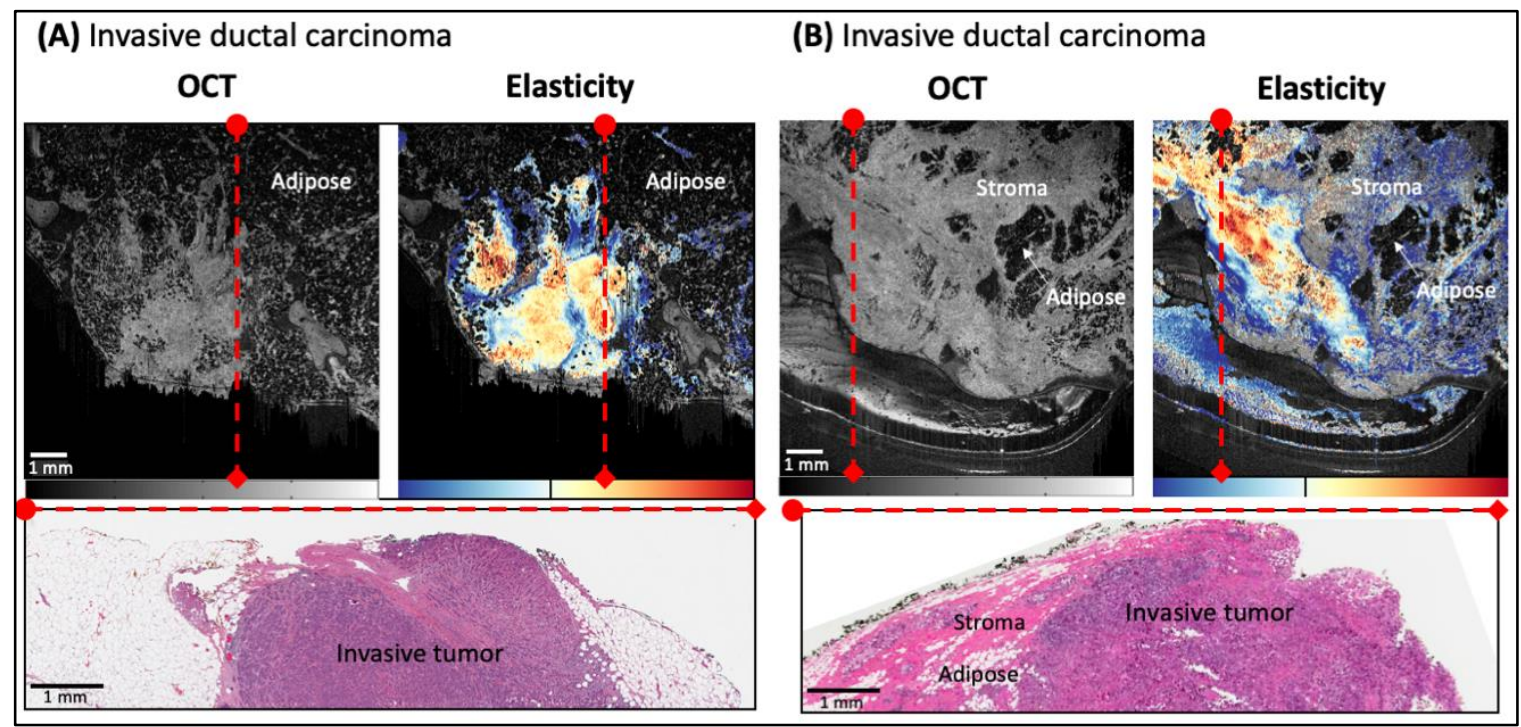

Figure 3. Example images of invasive ductal carcinoma. OCT, QME, and H\&E histology of (A) highly cellular invasive ductal carcinoma on the margin and (B) invasive ductal carcinoma on the margin surrounded by adipose and stroma tissues. Colorbars: OCT 0-40 dB; Elasticity 3.63-363 $\mathrm{kPa}$.

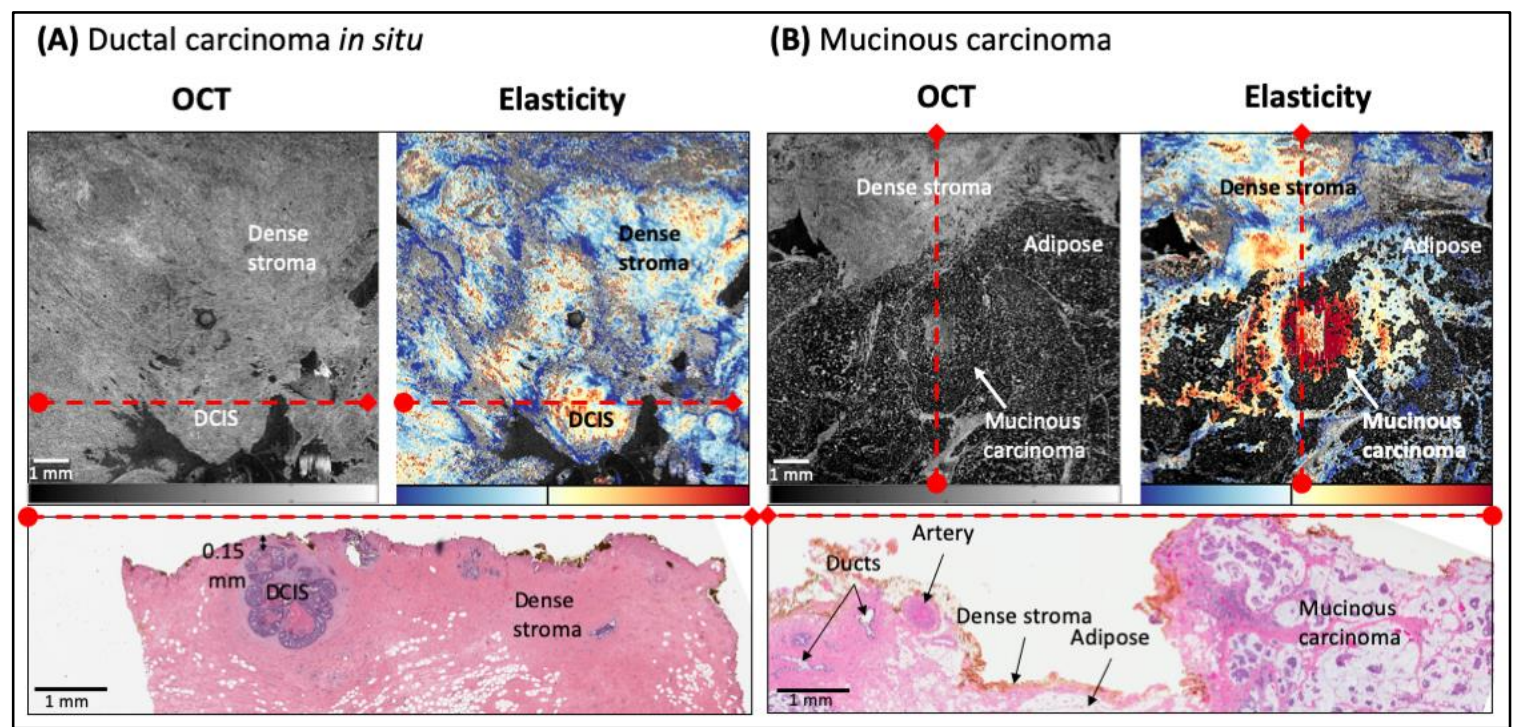

Figure 4. Example images of ductal carcinoma in situ (DCIS) and mucinous carcinoma. OCT, QME, and H\&E histology of (A) DCIS $0.15 \mathrm{~mm}$ from the margin and (B) invasive mucinous carcinoma present on the margin. Colorbars: OCT 0-40 dB; Elasticity 3.63-363 kPa. 